\title{
Identification of a novel crizotinib-sensitive BCL11A-ALK gene fusion in a nonsmall cell lung cancer patient
}

To the Editor:

Until now, more than 10 different ALK fusion partners have been discovered in nonsmall cell lung cancer (NSCLC): EML4, KIF5B, KLC1, TFG, TPR, HIP1, STRN, DCTN1, SQSTM1 and BIRC 6, among others $[1,2]$. We report here a novel ALK fusion partner in a NSCLC patient who responded well to the ALK inhibitor crizotinib.

A 64-year-old Chinese woman without a history of smoking was seen in the PLA General Hospital, Beijing, China in May 2016 because of cough and dyspnoea. Thoracic computed tomography (CT) showed a mass in the right upper lobe, multiple enlarged mediastinal lymph nodes and pleural effusion of right chest (figure 1a). After the pathological diagnosis of lung adenocarcinoma was established via endobronchial ultrasound guided-transbronchial needle aspiration, the Oncomine Focus Assay (OFA, Thermo Fisher Scientific, San Francisco, CA, USA) was used for hotspot mutations and gene fusion detection. High-throughput sequencing was performed on Ion PGM System (Thermo Fisher Scientific, Waltham, MA, USA). We achieved 149920 DNA sequencing reads and 274552 RNA sequencing reads, respectively. All the sequencing reads were aligned to the human genome (hg19), and the reads mapped to ALK $5^{\prime}$ and $3^{\prime}$ regions were utilised for ALK unbalanced $5^{\prime} / 3^{\prime}$ expression analysis. There were only seven RNA sequencing reads mapped to ALK $5^{\prime}$ region while 10366 reads were mapped to $3^{\prime}$ region. The $3^{\prime} / 5^{\prime}$ imbalance value is 0.06 , greater than the threshold value of 0.015 , which indicates a strong evidence of a fusion, and negative results were obtained while mapping sequencing reads to known fusion partner genes.

Owing to the presence of a novel ALK fusion, the ALK inhibitor crizotinib was prescribed tentatively at a dose of $250 \mathrm{mg}$ twice daily from June 2016. After 1 month treatment of crizotinib, the symptoms improved and the chest CT demonstrated partial response of the disease (figure 1b). Up to December 2016, the patient still remains on the crizotinib treatment without disease relapse.

Moreover, rapid amplification of cDNA ends (RACE) was accomplished using SMARTer RACE 5'/3' Kit (Takara, Mountain View, CA, USA) to obtain ALK fusion sequence, and we got the $1011 \mathrm{bp}$ sequence across the fusion site. Sequence analysis revealed that this patient had a novel BCL11A-ALK fusion (figure 1c).

BCL11A (B-Cell CLL/Lymphoma 11A), located on human chromosome 2, was known to play a role in B-cell malignancies and implicated in several biological processes including B-lymphopoiesis, neurogenesis and erythropoiesis [3]. Because this NSCLC patient, whose cancer harboured a novel BCL11A-ALK gene fusion, showed a partial response to ALK inhibitor crizotinib, BCL11A-ALK could be considered as an oncogenic fusion gene, which should be added to the list of ALK fusion genes. Next generation sequencing has displayed impressive capability to detect fluorescence in situ hybridisation (FISH)-negative ALK-rearranged NSCLC [4] and evolved to be a clinically practical alternative for diagnosis of ALK rearrangements besides of FISH, immunohistochemistry and RT-PCR based techniques. This study warrants future investigation of clinical characteristics and biological features of lung cancer harbouring BCL11A-ALK fusion gene.

@ERSpublications

We report a novel ALK fusion partner in a NSCLC patient who responded well to the ALK inhibitor crizotinib http://ow.ly/7O6f309KyFb

Cite this article as: Tian Q, Deng W-J, Li Z-W. Identification of a novel crizotinib-sensitive BCL11A-ALK gene fusion in a nonsmall cell lung cancer patient. Eur Respir J 2017; 49: 1602149 [https://doi.org/10.1183/ 13993003.02149-2016]. 

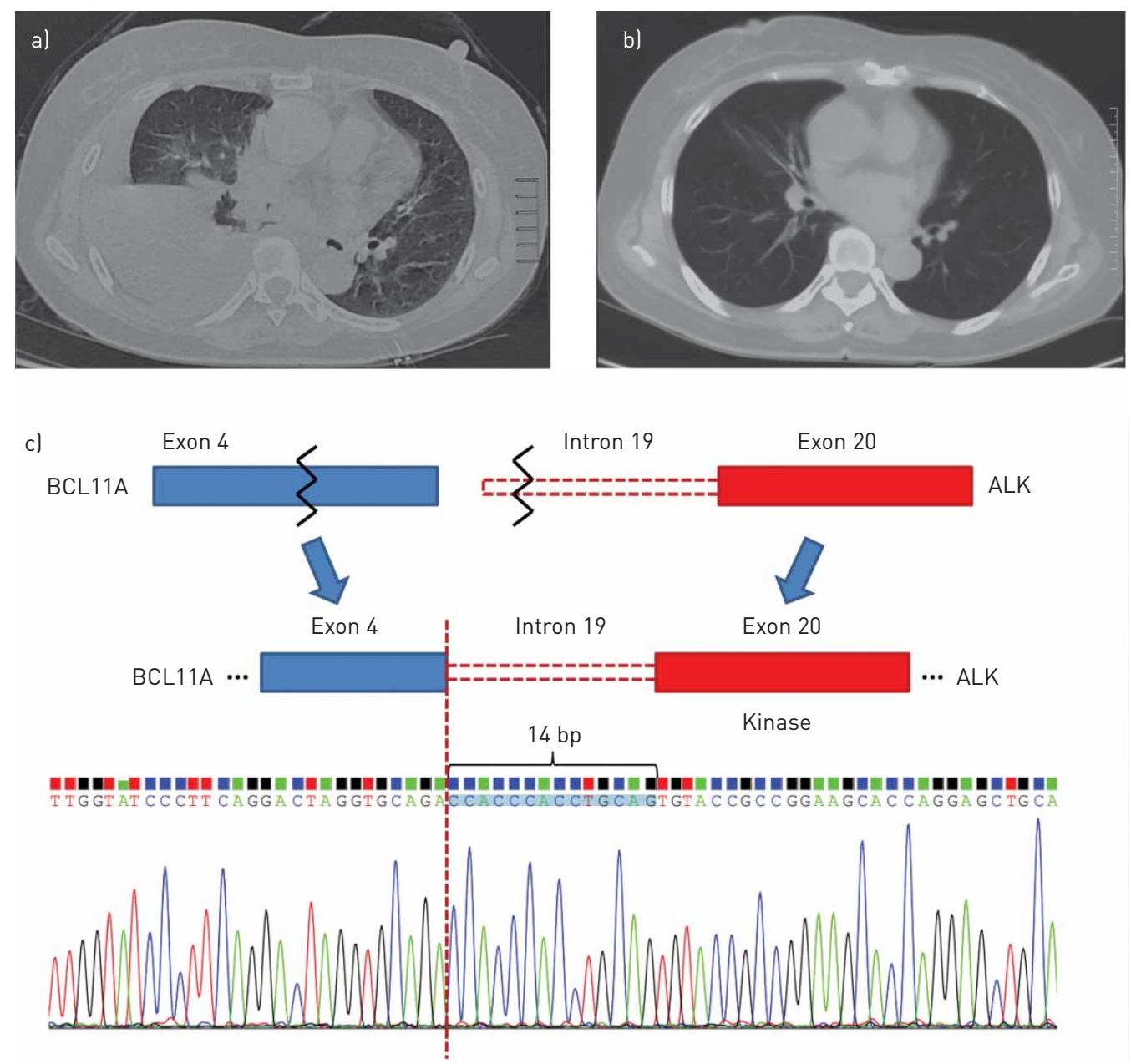

FIGURE 1 BCL11A-ALK fusion in nonsmall cell lung cancer. a) Thoracic computed tomography (CT) showed a mass in the right upper lobe, multiple enlarged mediastinal lymph nodes and pleural effusion of the right chest before crizotinib treatment. b) The mass and enlarged mediastinal lymph nodes and pleural effusion resolved 1 month after the crizotinib treatment as revealed by chest CT. c) A portion of BCL11A exon 4 (blue) fused to the last $14 \mathrm{bp}$ of ALK intron 19 (red) following exon 20. Validation of the fusion gene breakpoint using 5 '-RACE (rapid amplification of cDNA ends) is shown in the peak plot.

Qing Tian ${ }^{1}$, Wen-Jing Deng ${ }^{2}$ and Zong-Wei $\mathrm{Li}^{3}$

${ }^{1}$ Dept of Thoracic Surgery, PLA General Hospital, Beijing, China. ${ }^{2}$ Beijing GGT Co., Ltd, Beijing, China. ${ }^{3}$ Institute for Disease Control and Prevention, AMMS, Beijing, China.

Correspondence: Qing Tian, Dept of Thoracic Surgery, PLA General Hospital, Fuxing Road No. 28, Beijing 100853, China. E-mail: tianqing777@yeah.net

Received: Nov 022016 | Accepted after revision: Jan 032017

Conflict of interest: None declared.

\section{References}

Iyevleva AG, Raskin GA, Tiurin VI, et al. Novel ALK fusion partners in lung cancer. Cancer Lett 2015; 362: 116-121.

2 Shan L, Jiang P, Xu F, et al. BIRC6-ALK, a novel fusion gene in ALK break-apart FISH-negative lung adenocarcinoma, responds to crizotinib. J Thorac Oncol 2015; 10: e37-e39.

3 Bauer DE, Orkin SH. Hemoglobin switching's surprise: the versatile transcription factor BCL11A is a master repressor of fetal hemoglobin. Curr Opin Genet Dev 2015; 33: 62-70.

4 Ali SM, Hensing T, Schrock AB, et al. Comprehensive genomic profiling identifies a subset of crizotinib-responsive ALK-rearranged non-small cell lung cancer not detected by fluorescence in situ hybridization. Oncologist 2016; 21: $762-770$. 